\title{
Crianças hospitalizadas vítimas de acidentes na primeira infância
}

\author{
Children hospitalized for early childhood accidents
}

\author{
Niños hospitalizados víctimas de accidentes en la primera infancia
}

\section{Vanessa de Fatima Carvalho Simas ${ }^{1 *}$, Alessandra da Silva Souza ${ }^{2}$}

Como citar esse artigo. Simas, VFC; Souza, AS. Crianças hospitalizadas vítimas de acidentes na primeira infância. Revista Pró-UniverSUS. 2019 Jan./Jun; 10 (1): 25-28.

\section{Resumo}

Objetivo: Identificar os principais tipos de acidentes ocorridos na primeira infância no Hospital Universitário de Vassouras (HUV). Metodologia: Trata-se de uma pesquisa documental, de campo e com metodologia quantitativa. Foram analisados 50 prontuários dentro da faixa etária $(0$ a 6 anos) todos vitimas de acidentes. A coleta de dados foi realizada no período de julho a setembro de 2018 através de um instrumento de coleta. Resultados: A partir da análise de dados percebe-se que $68 \%$ dos acidentes foram devidos a quedas, $10 \%$ queimadura de $2^{\circ}$ grau, $10 \%$ ingestão de corpo estranho, $6 \%$ asfixia, $4 \%$ picada de escorpião e $2 \%$ intoxicação exógena. Os resultados demonstram que a maioria das vítimas, $60 \%$ são do sexo masculino. Diante destes dados é preciso que sejam tomadas medidas e realizados programas de prevenção de acidentes com crianças para que os acidentes diminuem. Conclusão: Conclui-se que o tema escolhido ajudou-nos a compreender as causas, consequências e a participação de cada um de nós na prevenção e promoção da saúde das crianças, que encontram-se em plena fase de crescimento e desenvolvimento. Desta forma, a enfermagem po $\neg$ derá desenvolver estratégias a partir dos dados expostos, como programas de extensão, enfocando fatores de risco, tomando medidas de segurança e prevenção envolvendo a criança, responsáveis e profissionais da enfermagem.

Palavras-chave: Acidentes da Primeira infância; Enfermagem; Hospitalização.

\begin{abstract}
Objective: To identify the epidemiological profile of children admitted to the University Hospital of Vassouras (HUV) victims of accidents in early childhood. Methodology: This is a documentary research, field and quantitative methodology. Fifty patient files within the age group ( 0 to 6 years) were analyzed, all victims of accidents. Data collection was performed from July to September 2018 through a collection instrument. Results: From the data analysis, $68 \%$ of the accidents were due to falls, $10 \%$ burns of the second degree, $10 \%$ foreign body ingestion, $6 \%$ asphyxia, $4 \%$ sting of scorpion and $2 \%$ exogenous intoxication. The results show that the majority of victims, $60 \%$ are males. Given these data, it is necessary to take measures and carry out programs to prevent accidents with children so that accidents decrease. Conclusion: We conclude that the theme chosen has helped us to understand the causes, consequences and participation of each of us in the prevention and promotion of children's health, which are in the midst of growth and development. In this way, nursing can develop strategies based on the data exposed, such as extension programs, focusing on risk factors, taking safety and prevention measures involving the child, caregivers and nursing professionals.
\end{abstract}

Keywords: Early Childhood Accidents; Nursing; Hospitalization.

\section{Resumen}

Objetivo: Identificar el perfil epidemiológico de niños internados en el Hospital Universitario de Vassouras (HUV) víctimas de accidentes en la primera infancia. Metodología: Se trata de una investigación documental, de campo y con metodología cuantitativa. Se analizaron 50 prontuarios dentro del grupo de edad ( 0 a 6 años) todos los accidentes. La recolección de datos se realizó en el período de julio a septiembre de 2018 a través de un instrumento de recolección. En el análisis de datos se observa que el $68 \%$ de los accidentes fueron debidos a caídas, $10 \%$ quemadura de $2^{\circ}$ grado, 10\% ingestión de cuerpo extraño, $6 \%$ asfixia, 4\% picada de escorpión y 2\% intoxicación exógena. Los resultados demuestran que la mayoría de las víctimas, el $60 \%$ son del sexo masculino. Ante estos datos es necesario que se toman medidas y programas de prevención de accidentes con niños para que los accidentes disminuyen. Conclusión: Se concluye que el tema escogido nos ha ayudado a comprender las causas, consecuencias y la participación de cada uno de nosotros en la prevención y promoción de la salud de los niños, que se encuentran en plena fase de crecimiento y desarrollo. De esta forma, la enfermería podrá desarrollar estrategias a partir de los datos expuestos, como programas de extensión, enfocando factores de riesgo, tomando medidas de seguridad y prevención involucrando al niño, responsables y profesionales de la enfermería.

Palabras clave: Accidentes de la Primera Infancia; Enfermería; Hospitalización. 


\section{Introdução}

Os acidentes na infância têm aumentando consideravelmente, representando atualmente um problema de Saúde Pública, podendo gerar incapacidade física temporária, sequelas ou até mesmo a morte em inúmeras crianças. Segundo o relatório da Organização Mundial de Saúde, a cada ano morre mais de 5 milhões de crianças vítimas de acidentes ${ }^{1}$.

A primeira infância é o período em que a criança consegue dominar a locomoção ereta até seu ingresso na escola, caracteriza-se por intensa atividade e pelas descobertas. É uma época de significativo desenvolvimento físico e da personalidade. $\mathrm{O}$ desenvolvimento motor prossegue continuamente. Nessa idade, a criança desenvolve a linguagem e estabelece relações sociais mais amplas, aprende os padrões de comportamento, adquire autocontrole e perícia, desenvolve uma crescente percepção de dependência e independência e começa a desenvolver o autoconceito ${ }^{2}$.

A Lei $\mathrm{n}^{\circ} 13.257$, de 8 de março de 2016, dispõe sobre as políticas públicas para a primeira infância e estabelece princípios e diretrizes para a formulação e a implementação de políticas públicas em atenção à especificidade e à relevância dos primeiros anos de vida no desenvolvimento infantil e no desenvolvimento do ser humano ${ }^{3}$.

Neste contexto podemos inferir que os primeiros anos de vida são fundamentais para o desenvolvimento do ser humano. O período da primeira infância é de grandes oportunidades para a plenitude da vida e também de muitas vulnerabilidades às influências e ações externas. Ao longo desse período as crianças desenvolvem suas habilidades motoras, emocionais, físicas, intelectual e social, porém enquanto esse processo não esta completo, elas ficam vulneráveis a uma série de perigos exigindo, cuidados especiais e atenção total.

AOrganização Mundial da Saúde define acidente como um acontecimento casual que independente da vontade humana, ocasionado por um fator externo originando dano corporal ou mental. São consideradas lesões não intencionais identificadas como afogamento, queda, queimadura, asfixia, acidente automobilístico e choque elétrico. Tais acidentes além de afetarem as crianças, afetam também suas famílias ${ }^{1}$.

A hospitalização infantil é um acontecimento estressante e traumatizante para a criança, pois é nesse período que ocorre uma ruptura com o seu meio social, suas atividades, hábitos e costumes. As crianças ficam imersas em um ambiente novo, cheio de restrições e rotinas, tendo à sua volta pessoas desconhecidas que passam a fazer parte do seu dia-a-dia, e, além disso, tem que se submeter a procedimentos que geram medo e dor. Isso requer do enfermeiro uma compreensão e avaliação mais minuciosa em relação à adaptação dessa criança ao ambiente hospitalar ${ }^{4}$.

Desta forma, tendo em vista a fragilidade do atendimento às crianças, o impacto destas ações na saúde infantil e a importância dos profissionais de saúde compreenderem as necessidades e direitos da criança e sua família, a fim de oferecer uma atenção humanizada e de qualidade, considera-se fundamental a assistência da equipe de enfermagem para realização da vigilância do desenvolvimento infantil.

Este estudo tem como objetivo identificar os principais tipos de acidentes ocorridos na primeira infância no Hospital Universitário de Vassouras (HUV).

\section{Metodologia}

Trata-se de uma pesquisa de campo, documental em prontuários e com metodologia abordagem quantitativa, buscando familiaridade com o problema em questão.

O cenário do estudo foi uma unidade pediátrica localizada em um hospital de ensino, no interior do Estado do Rio de Janeiro. O referido hospital é de médio porte, possui 20 leitos destinados a clientela pediátrica, sendo considerado como hospital de referencia para as localidades vizinhas. Este local foi escolhido por oferecer viabilidade para o estudo. Nessa perspectiva, solicitamos a autorização, por escrito, no Centro de Estudos do Hospital, oportunidade em que recebemos autorização para a coleta de dados no setor de pediatria.

Foram incluídos neste estudo os livros de registros de internação do setor de pediátrica dos anos de 2013 à 2018 , de todas as crianças de zero aos seis anos admitidas por acidentes, foram excluídos os registros que não se encontram nos critérios de inclusão.

A pesquisa foi submetida ao Comitê de Ética e Pesquisa da instituição conforme a resoluçãon ${ }^{\circ}$ 466/2012 do Conselho Nacional de Saúde - MS, que regulamenta as normas de Pesquisa envolvendo Seres Humanos. Foi aprovada sob o parecer de número 2.905.105 e CAAE 86374318.6.0000.5290.

Este artigo é parte integrante de um trabalho de conclusão de curso de Enfermagem da Universidade de Vassouras (UV).

\section{Resultados e Discussão}

Foram analisados 50 prontuários de crianças na primeira infância com registros de internação no setor de pediatria do HUV no período de Julho a Setembro de 2018.

Verificou-se que a prevalência dos acidentes na primeira infância no HUV ocorreu em crianças do sexo 
masculino perfazendo $60 \%$ da amostra, e $40 \%$ foram em crianças do sexo feminino, como pode ser observado na Tabela 1 a seguir:

Tabela 1. Distribuição segundo Sexo dos participantes ${ }^{12}$

\begin{tabular}{|c|c|c|}
\hline Sexo & Número de Clientes & Percentual (\%) \\
\hline Feminino & $\mathbf{2 0}$ & $\mathbf{4 0 \%}$ \\
\hline Masculino & $\mathbf{3 0}$ & $\mathbf{6 0 \%}$ \\
\hline Total & $\mathbf{5 0}$ & $\mathbf{1 0 0 \%}$ \\
\hline Fonte: Pesquisa do Autor, 2018 & & \\
\hline
\end{tabular}

Em um estudo realizado acerca desta problemática também foi verificado a prevalência de acidentes em crianças do sexo masculino. Esse resultado justifica-se, provavelmente, pelos diferentes comportamentos de cada sexo e por fatores culturais, que determinam maior vigilância sobre as meninas e maior liberdade para os meninos, isso tendo em conta a nossa realidade 5 .

Emrelaçãoàfaixaetáriadascriançashospitalizadas verificou-se que $46 \%$ dos acidentes ocorreram na fase pré escolar $(46 \%)$ que compreende a faixa etária de 3 a 6 anos, $44 \%$ ocorreram em lactentes que abrange as crianças entre 1 até 12 meses e $10 \%$ na fase neonatal, sendo do nascimento até 28 dias de vida. Como pode ser observado no Gráfico 1. A maior frequência de crianças nesta faixa etária pode ser entendida devido às crianças em geral possuírem uma enorme curiosidade com relação ao ambiente.

Gráfico 1. Distribuição segundo Faixa Etária dos participantes ${ }^{12}$

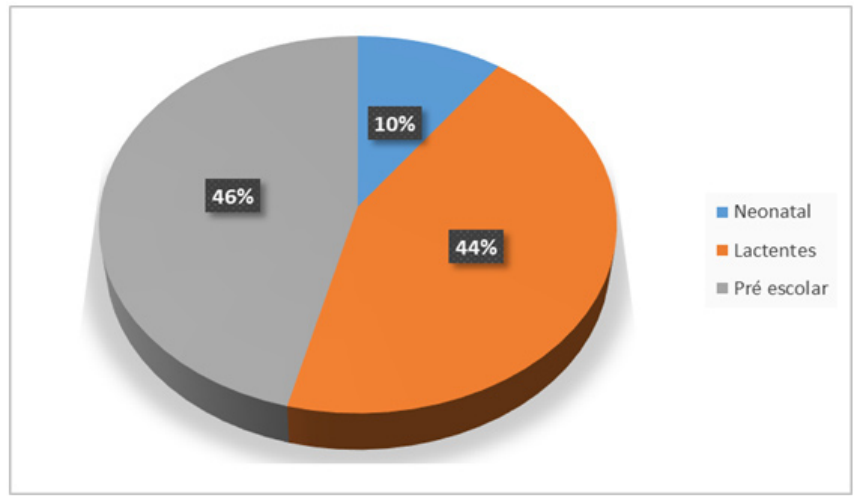

Fonte: Pesquisa do Autor, 2018

As crianças, no decorrer da infância, se encontram propensas a acidentes em virtude da sua imaturidade, curiosidade, intenso crescimento e desenvolvimento, sendo indefesas e vulneráveis aos fatores do ambiente que contribuem para os mesmos ${ }^{6}$.

Em relação aos tipos de acidentes, a amostra demonstrou que $68 \%$ das internações foram devido à queda com o diagnóstico de TCE (Traumatismo Cranioencefalico), $10 \%$ queimadura $2^{\circ}$ grau, $10 \%$ ingestão de corpo estranho, $6 \%$ asfixia, $4 \%$ picada de escorpião e $2 \%$ intoxicação exógena. Apresentado no
Gráfico 2 a seguir:

Gráfico 2. Distribuição segundo os tipos de acidentes ${ }^{12}$

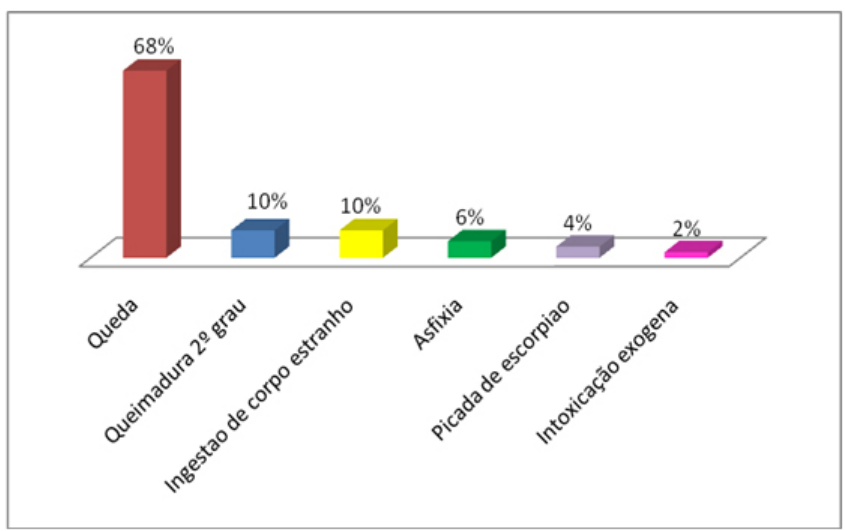

Fonte: Pesquisa do Autor, 2018

Neste sentido, foi constatadaumaalta porcentagem de quedas levando a hospitalização das crianças no HUV. Este fato explica-se provavelmente porque as crianças são ativas e não têm noção do perigo, sua curiosidade as impulsiona a investigar e a imitar o comportamento dos outros e por estar em fase de descobertas do meio ambiente, favorecendo a ocorrência deste evento na população infantil. Os traumatismos crânio-encefálico têm como principais causas as quedas, acidentes de trânsito e acidentes com bicicletas. O TCE pode ser definido como toda a lesão causada no sistema nervoso central que foi provocado por um trauma, afetando os ossos, crânio, couro cabeludo, cérebro, meninges, decorrendo de uma força mecânica? .

A segunda maior causa de hospitalização encontrada no estudo foram às queimaduras e a ingestão de corpo estranho. Muitas vezes as queimaduras são causadas por descuido dos pais ou cuidadores. Entre as causas mais comuns de queimaduras com crianças até seis anos de idade estão às decorrentes de escaldamentos (manipulação de líquidos quentes, como água fervente, pela curiosidade característica da idade) ${ }^{8}$.

A ingestão de corpos estranhos é um problema comum na população pediátrica, com até $75 \%$ dos casos ocorrendo com crianças menores de cinco anos de idade. Frequentemente as crianças levam à boca objetos que estão sendo investigados por elas e os objetos acabam sendo acidentalmente engolidos. Estima-se que até $40 \%$ das ingestões de corpos estranhos por crianças não são testemunhadas e por essa razão o número real de casos pode ser mais alto do que aqueles que são relatados? .

À asfixia é o primeiro risco apresentado à criança a partir do nascimento. Pode ocorrer principalmente durante o sono, onde: lençóis, travesseiro, bichinhos de pelúcia, roupa de cama e até mesmo as grades do berço associadas à roupa de cama folgada tornam-se fatores de risco eminentes. Em seguida vêm os pequenos objetos como: brinquedos, botões, enfeites, acessórios 
das roupinhas e até mesmo alimentos impróprios para a idade, são fatores facilitadores para a asfixia ${ }^{10}$.

Segundo o Ministério da Saúde, as notificações dos acidentes por animais peçonhentos chegam perto de 100 mil por ano e vem aumentando gradativamente, expondo o número de casos e mortalidade por tipo de ocorrências, entre eles tendo o escorpião 37.495 casos e $2 \%$ mortalidade, desempenhando um problema de saúde pública desinente da elevada freqüência e da gravidade. As picadas de escorpião destacam-se entre os acidentes com animais peçonhentos na infância, devido às particularidades de vida desses artrópodes e as freqüentes exposições das crianças. Nessa faixa etária, existe o risco de maior gravidade ${ }^{11}$.

Por fim, mas não menos grave há a intoxicação tanto por produtos de higiene e limpeza como medicações mal armazenados, são os mais rotineiros acidentes nesta primeira fase da infância. O armazenamento desses produtos em locais baixos ou sem travas podem ser uma combinação propícia para a ocorrência do acidente. Porém vale ressaltar que com a devida orientação são facilmente evitados ${ }^{10}$.

Neste contexto, a vulnerabilidade da criança mediante ao novo deve ser considerada o principal fator de risco e de ocorrência de acidentes. Enfatiza-se a necessidade de capacitação da equipe de enfermagem para assistir a criança, pois a mesma apresenta necessidades diferenciadas conforme o período de crescimento e desenvolvimento que se encontra. A assistência à criança exige conhecimento teóricoprático, agilidade, habilidade, segurança, calma para atender de forma humanizada a criança e sua família ${ }^{11}$.

\section{Conclusão}

Os acidentes têm-se revelado como uma das principais causas dos atendimentos, hospitalização, incapacidades e óbitos em crianças, nos vários países e tem contribuído, de forma considerável, para manter elevada a taxa de mortalidade infantil.

Entende-se que o tema escolhido foi de muita importância pois ajudou-nos a compreender os principais tipos de acidentes ocorridos em crianças na primeira infância que encontram-se em plena fase de crescimento e desenvolvimento. Pode-se dizer que conseguimos atingir os objetivos delineados para o estudo.

De acordo com dados verificados neste estudo, foi possível descrever o perfil da amostra da seguinte forma: $60 \%$ dos acidentes ocorreram no sexo masculino, $46 \%$ das crianças hospitalizadas estavam na fase pré escolar, que compreende a faixa etária de 3 a 6 anos, e $68 \%$ dos acidentes foram as quedas, seguidos de queimadura de $2^{\circ}$ grau (10\%), ingestão de corpo estranho (10\%), asfixia (6\%), picada de escorpião (4\%) e 2\% intoxicação exógena.

Consideramos que estes dados são importantes e poderão subsidiar o planejamento e implantação de medidas preventivas com o intuito de reduzir o impacto desse agravo na saúde da população infantil. Dessa forma, a enfermagem poderá desenvolver estratégias a partir dos dados expostos, como programas de extensão, com foco nos fatores de risco, tomando medidas de segurança e prevenção de acidentes envolvendo a criança, sendo estendido aos responsáveis e os profissionais de enfermagem.

Neste contexto, o tema precisa ser amplamente discutido na sociedade, e de modo especial no ensino de graduação de cursos das áreas da saúde, contribuindo para reflexão sobre a necessidade de adotar um comportamento preventivo para minimizar os casos de acidentes em crianças na primeira infância.

\section{Referências Bibliográficas}

1. OMS. Organização Mundial da Saúde. "Saúde 21". Uma introdução ao enquadramento político da saúde para todos na Região Europeia da OMS. 2012 .

2. Wong HMJ, Wilson D. Fundamentos de Enfermagem Pediátrica. Rio de Janeiro, 10. Ed: Elsevier, 2018.

3. Brasil. Plano Nacional da Primeira Infância. Projeto Observatório Nacional da primeira Infância. Mapeamento da Ação Finalística Evitando Acidentes na Primeira Infância. 2014

4. Sousa LD, et al. A família na unidade pediátrica: Percepções da equipe de enfermagem acerca da dimensão cuidadora. Cienc. Enferm., 2011;17(2):87-95.

5. Paes CEN, Gaspar VLV. As Injúrias não intencionais no âmbito domiciliar: a casa segura. J Pediat, 2005;81(5, supl.).

6. Lima EJF. Pediatria ambulatorial. Rio de Janeiro: MED BOOKEditora Científico. 2008

7. Almeida J, Lima M, Silva R. Acidentes Domésticos na Infância. 2013. Trabalho de Conclusão de Curso. Escola Superior de Saúde. Licenciatura em Enfermagem, Universidade do Mindelo (UM). Mindelo, 2013. Disponível em: http://portaldoconhecimento.gov.cv/handle/10961/2488

8. Oliveira M, Parolin F, Teixeira J. Trauma-Atendimento PréHospitalar.3.ed. São Paulo: Atheneu Rio, p. 285-287, 2014.

9. Carvalho BCN, Talini C, Antunes LA, Aranha Junior AA, Alves ALF, Almeida GC. Ingestão de corpo estranho (prego) por crianças - manejo conservador. Relatos Casos Cir.2017;(2):1-3.

10. Pereira SFA, Garcia CA. Prevenção de acidentes domésticos na infância. Rev Enferm UNISA. 2009[citado em 2015 jan. 16];10(2):172-7.

11. Brasil. Ministério da Saúde. Secretaria de Atenção à Saúde. Departamento de Atenção Básica. Vigilância em saúde: zoonoses. Secretaria de Atenção à Saúde, Departamento de Atenção Básica. Brasília: Ministério da Saúde, 2009

12. Simas VFC. Perfil de crianças hospitalizadas na pediatria vítimas de acidentes na primeira infância. Trabalho de Conclusão de Curso. Universidade de Vassouras. Rio de Janeiro. 2018

13. Cunha LNGC, Souza AS. Análise Bibliográfica acerca da Assistência de Enfermagem em situações de emergência pediátrica. Revista PróUniversus, 2013; 4(2) 